\title{
Das Basler Interview für Psychosen (BIP): Struktur, Reliabilität und Validität
}

\section{The Basel Interview for Psychosis (BIP): Structure, Reliability and Validity}

Autoren

Institute
A. Riecher-Rössler' ${ }^{1}$, T. Ackermann ${ }^{1}$, M. Uttinger ${ }^{1}$, S. Ittig ${ }^{1}$, S. Koranyi ${ }^{2}$, C. Rapp ${ }^{3}$, H. Bugra ${ }^{1}$, E. Studerus ${ }^{1}$

Zentrum für Gender Research und Früherkennung, Universität Basel, Universitäre Psychiatrische Kliniken, Basel

2 Institut für Psychosoziale Medizin und Psychotherapie, Universitätsklinikum Jena

Klinisch Psychologischer Dienst, Psychiatrische Dienste, Solothurn
Schlüsselwörter

- Klinisches Interview

- Psychose

- Schizophrenie

- Früherkennung

Key words

- clinical interview

psychosis

- schizophrenia

- early detection

\section{Bibliografie}

Dol http://dx.doi.org/ 10.1055/s-0034-1398999 Fortschr Neurol Psychiatr 2015; 83: 99-108 ๑ Georg Thieme Verlag KG Stuttgart · New York . ISSN 0720-4299

Korrespondenzadresse
Prof. Anita Riecher-Rössler
Zentrum für Gender Research
und Früherkennung, Universität
Basel, Universitäre
Psychiatrische Kliniken
Kornhausgasse 7
4051 Basel
Schweiz
anita.riecher@upkbs.ch

Korrespondenzadresse Prof. Anita Riecher-Rössler Basel, Universitäre Psychiatrische Kliniken Kornhausgasse 7

anita.riecher@upkbs.ch

\section{Zusammenfassung \\ $\nabla$}

Hintergrund: Obwohl in den letzten Jahren zahlreiche Instrumente entwickelt worden sind, um Psychoserisikopersonen und Psychoseersterkrankte zu identifizieren, gab es bisher keine geeigneten Instrumente, um solche Personen ausführlicher über ihren Krankheitsverlauf sowie die Risikofaktoren und frühen Indikatoren der Erkrankung zu befragen. Wir haben deshalb das Basler Interview für Psychosen (BIP) entwickelt. Ziel der vorliegenden Arbeit ist es, die Entwicklung und den Aufbau des BIP zu beschreiben sowie über seine psychometrischen Eigenschaften zu berichten.

Methoden: Das BIP ist ein halbstrukturiertes klinisches Anamneseinterview, das im Rahmen des Basler Projekts zur Früherkennung von Psychosen (FePsy) auf der Basis der wichtigsten in der Literatur beschriebenen Risikofaktoren und Indikatoren für Psychosen und unter Berücksichtigung schon existierender Fragebögen entwickelt wurde. Es gliedert sich in sechs Bereiche: 1. Soziale und körperliche Entwicklung/Familie, 2. Anzeichen und Symptome, 3. Vulnerabilität, 4. Hilfesucheverhalten, 5. Krankheitseinsicht, 6. Interviewereinschätzung. Um die Interrater-Reliabilität der Items der Abschnitte 2 und 3 zu bestimmen, wurden 20 Patienten von insgesamt 8 unterschiedlichen Ratern beurteilt. Die faktorielle Struktur des Abschnitts 2.3 „Prodromi und Symptome“ wurde in einer Stichprobe von 120 Psychoserisikopersonen und 77 Erstpsychosepatienten untersucht. Auf der Basis der gefundenen Faktorstruktur wurden neue Skalen gebildet und auf ihre Reliabilität und Validität untersucht.

Ergebnisse: Von 153 untersuchten Items der Abschnitte 2 und 3 wurden 150 (98\%) mit ausreichend hoher Übereinstimmung (Interrater-Reliabilität >0,4) eingeschätzt. Die Items des Abschnitts 2.3 „Prodromi und Symptome“ konnten zu 5 Subskalen gruppiert werden. Die Subskalen wiesen überwiegend gute bis sehr gute interne Konsisten-

\section{Abstract \\ $\nabla$}

Background: Although several instruments have been developed to identify patients with an atrisk mental state (ARMS) for psychosis and first episode of psychosis (FEP), up to now there were no instruments for a detailed assessment of risk factors and indicators of emerging psychosis and the temporal development of psychiatric symptoms over the whole life span in these patients. We therefore developed the Basle Interview for Psychosis (BIP). The aim of this study is to describe the development of the BIP and to report about its psychometric properties.

Methods: The BIP is a comprehensive semi-structured interview that was developed for the Basel early detection of psychoses (FePsy) study. Its items were derived from the most important risk factors and indicators of psychosis described in the literature and from several existing instruments. It contains the following six sections: 1 ) social and physical development and family, 2) signs and symptoms, 3) vulnerability, 4) help-seeking behavior, 5) illness insight, 6) evaluation of the interview. To estimate the inter-rater reliabilities of the items of sections 2 and 3, 20 interviews were conducted and rated by 8 well-trained raters. The factorial structure of the BIP section "signs and symptoms" was explored in a sample of 120 ARMS and 77 FEP patients. On the basis of the discovered factorial structure, we created new subscales and assessed their reliabilities and validities. Results: Of the 153 studied items of sections 2 and 3, 150 (98\%) were rated with sufficiently high agreement (inter-rater reliability $>0.4$ ). The items of section "signs and symptoms" could be grouped into 5 subscales with predominantly good to very good internal consistencies, homogeneities, and discriminant and convergent validities. Predictive validities could be demonstrated for the subscales "Positive Psychotic Symptoms", "Disturbance of Thinking" and the total score. 
zen, Homogenitäten und diskriminante und konvergente Validitäten auf. Für die Subskalen „Positive psychotische Symptome“ und „Störungen des Denkens“ sowie den Gesamtscore konnte zudem eine prädiktive Validität nachgewiesen werden.

Diskussion: Mit dem BIP haben wir erstmals ein halbstrukturiertes klinisches Anamneseinterview konstruiert und validiert, das geeignet ist, Psychoserisikopatienten und Psychoseersterkrankte detailliert über Psychoserisikofaktoren und -indikatoren sowie den psychopathologischen Verlauf seit der Kindheit zu befragen. Wir konnten zeigen, dass das BIP hervorragende psychometrische Eigenschaften aufweist.
Discussion: The BIP is the first interview for comprehensively assessing risk factors and indicators of emerging psychosis and the temporal development of psychiatric symptoms over the whole life span, which has been validated in ARMS and FEP patients. We could show that the BIP has excellent psychometric properties.

\section{Einleitung \\ $\nabla$}

Die Früherkennung und Frühbehandlung von Psychosen ist in den letzten 20 Jahren zunehmend in den Fokus des wissenschaftlichen und klinischen Interesses gerückt. Im Zuge dieser Entwicklung sind zahlreiche Kriterien und Instrumente entstanden, die es erlauben, das Risiko für eine Psychose bereits während eines potenziellen Prodromalstadiums reliabel und valide einzuschätzen (für eine Übersicht siehe [1]).

Zur Erfassung eines eher psychosefernen Risikostadiums haben sich die ursprünglich von Huber und Gross [2] in den 1960er Jahren beschriebenen und später von Klosterkötter et al. [3] prospektiv validierten selbstwahrgenommenen Beeinträchtigungen im Denken und der Wahrnehmung, sog. Basissymptome, durchgesetzt, während für spätere, psychosenähere Risikostadien heutzutage zumeist die ursprünglich in Melbourne entwickelten UltraHigh-Risk(UHR)-Kriterien verwendet werden [4]. Ersteres Stadium wird häufig durch das Schizophrenia Proneness Instrument, Adult Version (SPI-A) [5], operationalisiert, während für die Erfassung von letzterem das Structured Interview for Prodromal Symptoms (SIPS) [6] und das Comprehensive Assessment of At-Risk Mental State (CAARMS) [7] international am meisten verbreitet sind. In Basel haben wir im Rahmen der Studie zur Früherkennung von Psychosen (FePsy) [8 - 10] das sog. Basel Screening Instrument für Psychosen (BSIP) entwickelt, das neben den UHR-Kriterien auch noch eine weitere Risikokategorie enthält, die durch unspezifische Risikofaktoren operationalisiert wird [11].

Ein Nachteil all dieser Instrumente ist jedoch, dass sie nur die Risikokriterien abfragen und nicht dazu geeignet sind, ein breites Spektrum der in der Literatur beschriebenen Risikofaktoren und -indikatoren abzubilden oder den psychopathologischen Verlauf seit der Kindheit zu erfassen. Zwar existiert mit dem Interview für die retrospektive Erfassung des Erkrankungsbeginns und -verlaufs bei Schizophrenie und anderen Psychosen (IRAOS) [12], das im Rahmen der Mannheimer ABC-Studie [13] entwickelt und validiert wurde, bereits ein Instrument, das den psychopathologischen Verlauf bei Psychoseersterkrankten retrospektiv erfassen kann, jedoch eignet sich dieses nicht für die Anwendung bei Psychoserisikopatienten. Um sowohl Psychoserisikopersonen als auch Psychoseersterkrankte nach dem Screening mit dem BSIP detaillierter befragen zu können, haben wir deshalb ein umfassendes, halbstrukturiertes Interview, das sog. Basler Interview für Psychosen (BIP), entwickelt.

Das BIP ist seit dem Jahr 2000 in Gebrauch und wurde in bisher publizierten Studien u.a. dazu verwendet, die frühesten selbstwahrgenommenen Anzeichen einer psychischen Erkrankung [14], aktuellen und früheren Konsum von Cannabis [15, 16], die Dauer der unbehandelten Psychose bzw. Erkrankung $[17,18]$ so- wie Behandlungswege [17] bei Psychoserisiko und Psychoseersterkrankten zu erfassen.

Ziel der vorliegenden Arbeit ist es, die Entwicklung und den Aufbau des BIP zu beschreiben sowie über seine Anwendbarkeit, Interrater-Reliabilität, faktorielle Struktur und Validität zu berichten.

\section{Methoden \\ $\nabla$}

Entwicklung und Aufbau des Basler Interviews für Psychosen (BIP)

Das BIP ist ein halbstrukturiertes klinisches Anamneseinterview, das im Rahmen der Basler FePsy-Studie [8-10] entwickelt wurde, um Patienten, die mit dem BSIP [11] als Psychoserisiko- oder Erstpsychosepatienten identifiziert wurden, ausführlicher über ihre Krankheitsgeschichte und ihre Risikofaktoren für Psychosen zu befragen. Das BIP enthält Items aus 6 Bereichen ( $\bullet$ Abb. 1). In seinem Aufbau und Inhalt lehnt es sich an die ursprüngliche Version des IRAOS [12] an. Als Grundlage für die Konstruktion der BIP-Items dienten - neben dem IRAOS - die wichtigsten in der Literatur genannten Risikofaktoren und frühen Anzeichen einer psychotischen Erkrankung sowie der Explanatory Model Interview Catalogue (EMIC) [19], das Interview zur Erfassung der Krankheitsbewältigung (IKB) [20], die Bonner Skala für die Beurteilung von Basissymptomen (BSAPS) [21] und die Scale of Prodromal Symptoms (SOPS) [22].

Die Durchführung des BIP dauert ca. 90 Min. Für die Bewertung der Items wird nicht nur das direkte Gespräch mit dem Patienten berücksichtigt, sondern auch sämtliche anderen kurzfristig verfügbaren Informationsquellen, wie etwa Arztberichte, Fremdanamnesen, Zuweisungsschreiben und mündliche Berichte von Angehörigen. Dies ist insbesondere für äußerlich feststellbare Veränderungen im Verhalten sowie die genaue zeitliche Datierung von Symptomen und einschneidenden Lebensereignissen wichtig.

Im ersten Abschnitt des BIP werden die soziale und körperliche Entwicklung des Patienten, seine berufliche und familiäre Einbindung sowie sein sozialer Status erfasst. Gefragt wird nach schulischer und beruflicher Entwicklung, insbesondere ob Abschlüsse im üblichen Alter erreicht werden konnten, d.h., ob es wegen Schwierigkeiten zu Unterbrechungen und Abbrüchen der Ausbildung, einem Wechsel der Schule bzw. Ausbildungsstätte oder zu einem späteren beruflichen Abstieg gekommen ist. Zusätzlich können durch Schwierigkeiten verursachte Veränderungen in Partnerschaft, Familie und der Lebenssituation, frühere somatische Krankheiten mit möglicher Auswirkung auf das Gehirn, Auffälligkeiten in der frühkindlichen Entwicklung, Drogenkonsum und die genetische Vorbelastung durch psychische Krank- 
1. Soziale und körperliche Entwicklung, Familie

1.1 Schulbildung

1.2 Berufsausbildung

1.3 Berufstätigkeit

1.4 Partnerschaft und Kinder

1.5 Lebensverhältnisse und Wohnsituation

1.6 Körperliche Krankheiten und Entwicklungsstörungen

1.7 Drogenkonsum

1.8 Familie und Gesundheit

2. Anzeichen und Symptome

2.1 Anlass der Konsultation

2.2 Früheste Anzeichen einer psychischen Erkrankung

2.3 Prodromi und Symptome

2.3.a Sorgen, Unruhe, Ängste (3)

2.3.b Körperliche Beschwerden (5)

2.3.c Denken, Konzentration (7)

2.3.d Zwänge (1)

2.3.e Stimmung, Fühlen (9)

2.3.f Sensibilität, Misstrauen (3)

2.3.g Sozialer Rückzug, Verhaltensänderung (2)

2.3.h Übernatürliches, Unerklärliches (3)

2.3.i Derealisation, Depersonalisation, Wahrnehmungsstörungen (5)

2.3.k Halluzinationen (5)

2.3.I Wahn (5)

2.3.m Gedankeneingabe, Gedankenlautwerden, Gedankenentzug (3)

2.3.n Fremdbeeinflussung (1)

2.3.o Soziale Anpassung, Behinderung (10)

\section{Vulnerabilität}
3.1 Kritik oder Ablehnung
3.2 Konflikte
3.3 Intensive positive Gefühle
3.4 Unklare Situationen
3.5 Laute Umgebung
3.6 Arbeiten unter Zeitdruck

\section{Hilfesuchverhalten}

5. Krankheitseinsicht

6. Intervieweinschätzung

Anhang
Abb. 1 Aufbau des Basler Interviews für Psychosen (BIP). Für den Abschnitt 2.3. (Prodromi und Symptome) ist in Klammern die Anzahl der Items aufgeführt. heiten in der Familie erhoben werden. Durch die Erfassung von Unterbrechungen oder Verzögerungen in der sozialen Entwicklung kann ein „Knick in der Lebenslinie“ erkannt und datiert werden.

Der zweite Abschnitt des Interviews dient der Erfassung von frühen Anzeichen und Symptomen. In den Abschnitten 2.1 und 2.2 werden die Gründe der aktuellen Konsultation, bisherige Behandlungswege sowie Zeitpunkt und Art der frühesten Anzeichen einer psychischen Erkrankung erfragt. Im Abschnitt 2.3 werden psychopathologische Symptome erfasst, die typischerweise in der Prodromalphase einer schizophrenen Psychose vorkommen. Das Auftreten dieser Symptome kann anhand folgender Antwortmöglichkeiten eingeschätzt werden: (a) „nie vorhanden“, (b) „schon immer vorhanden“ oder (c) „neu aufgetreten nach dem 12. Lebensjahr“. Ist ein Symptom nach dem 12. Lebensjahr neu aufgetreten, muss vom Interviewer genau eingeschätzt werden, wann dies erstmals der Fall war. Die Symptome sind inhaltlich in 14 Symptomgruppen aufgeteilt. Als repräsentativ für alle Symptome einer Symptomgruppe kann das früheste Symptom der Gruppe markiert werden. Für dieses erste Symptom sollen auf einer dreistufigen Skala sein Verlauf und die aktuelle Ausprägung eingeschätzt werden.
Im dritten Teil des Interviews wird die Vulnerabilität des Patienten erfasst. Dem Patienten werden mehrere Situationen genannt, die psychologisch belastend sein können. Der Patient soll angeben, ob er sich durch die jeweilige Situation belastet fühlt, ob dies schon immer so war und ob sich seine Beschwerden dadurch verschlimmern. Im vierten und fünften Abschnitt werden das Hilfesuchverhalten, frühere Therapien und die subjektive Krankheitseinsicht des Patienten erhoben. Im sechsten Abschnitt können nach dem Interview die Krankheitseinsicht des Patienten, die Angemessenheit der gegebenen Informationen, das äußere Erscheinungsbild und das Verhalten des Patienten eingeschätzt werden.

Ein Zeitraster ermöglicht es dem Interviewer, die psychopathologischen Auffälligkeiten in Verbindung mit den wichtigsten Lebensereignissen des Patienten chronologisch einzuordnen. Dies dient dazu, den Beginn der psychopathologischen Veränderung zeitlich genau einschätzen zu können.

\section{Interrater-Reliabilitätsstudie}

Um die Interrater-Reliabilität der BIP-Items der Abschnitte 2 und 3 zu bestimmen, wurden 20 Patienten von insgesamt 8 unterschiedlichen Ratern beurteilt. Bei 19 Patienten erfolgte die Beur- 
teilung auf der Basis von auf Video aufgezeichneten Interviews; ein Patient wurde direkt während des Interviews beurteilt. 12 der 20 gerateten Patienten wurden von jeweils 7 Ratern beurteilt, 3 Patienten von 6 Ratern, 3 Patienten von 5 Ratern, 1 Patient von 4 Ratern und 1 Patient von 3 Ratern. Um trotz unterschiedlicher Anzahl von Ratern eine genaue Schätzung der Interrater-Reliabilitäten zu gewährleisten, wurden statistische Analysemethoden verwendet, die damit umgehen können (siehe unten). Alle Patienten gaben ihr schriftliches Einverständnis zur Teilnahme an der Studie und gegebenenfalls zur Videoaufzeichnung des BIP-Interviews.

\section{Stichproben}

Die Stichprobe der Patienten umfasste 20 Personen, die im Rahmen der FePsy-Sprechstunde abgeklärt wurden. 12 dieser Patienten waren anhand des BSIP als Psychoserisikopatienten und 8 als Ersterkrankte identifiziert worden. Die Patienten waren zwischen 18 und 40 Jahre alt $(M=25,4 ; S D=6,7)$ und zu $75 \%$ Männer.

Die Stichprobe der Rater bestand aus 7 Psychologinnen und 1 Psychologen des FePsy-Projekts. Davon waren 4 Doktorandinnen, 3 Postdocs und 1 Masterstudentin. Alle Rater hatten klinische Erfahrung mit der Patientenklientel der FePsy-Sprechstunde und im Vorfeld ein 10-stündiges videogestütztes BIP-Training absolviert.

\section{Statistische Analyse}

Für die Berechnung der Interrater-Reliabilitätskoeffizienten wurde das Programm AgreeStat 2011.3 [23] verwendet. Bei nominalskalierten Items wurden die Interrater-Reliabilitäten anhand des AC1-Koeffizienten [24] geschätzt. Dieser hat gegenüber dem traditionell häufiger verwendeten Cohen's Kappa den Vorteil, dass er einerseits robust gegenüber Verzerrungen durch ungleich hohe Beobachtungsprävalenzen ist [vgl. 25] und andererseits mit fehlenden Werten umgehen kann. Die Konfidenzintervalle der AC1-Koeffizienten wurden so bestimmt, dass die InterraterReliabilitäten repräsentativ für die Population der Rater und der Population der Patienten sind.

Bei intervallskalierten Items wurden die Interrater-Reliabilitäten anhand der Intraklassenkorrelation $\operatorname{ICC}(2,1))$ nach der Methode von Gwet [26] geschätzt, da diese im Unterschied zur ursprünglichen Methode von Shrout und Fleiss [27] mit fehlenden Werten umgehen kann.

Zur Interpretation der Interrater-Reliabilitätskoeffizienten wurden die Kriterien von Landis und Koch verwendet [28]: 0 $0,20=$ schwache Übereinstimmung, $0,21-0,40=$ leichte Übereinstimmung, $0,41-0,60=$ mittlere Übereinstimmung, $0,61-0,80$ starke Übereinstimmung, $0,81-1,00=$ beinahe vollkommene Übereinstimmung.

\section{Studie zur faktoriellen Struktur und Konstruktion} neuer Subskalen

Stichprobe

Für die Bestimmung der faktoriellen Struktur der 62 Items des BIP-Abschnitts 2.3 (Prodromi und Symptome) wurden die BIPDaten aller Personen verwendet, die zwischen dem 1. März 2000 und dem 28. Februar 2014 als Psychoserisikopatienten oder Psychoseersterkrankte in die FePsy-Studie eingeschlossen worden waren und bei denen der Abschnitt 2.3 des BIP mindestens zur Hälfte vollständig erhoben worden war.

Von den insgesamt 253 Patienten, die in dieser Zeit in die FePsyStudie eingeschlossen worden waren, hatten 197 Personen ausreichend vollständig erhobene BIP-Fragebögen. Personen mit und ohne ausreichend vollständig erhobene BIP-Fragebögen unterschieden sich nicht signifikant bzgl. Geschlecht, Alter und Anzahl Bildungsjahre, was darauf hindeutet, dass die Gruppe von FePsy-Patienten, die für die Faktoranalyse verwendet werden konnte, repräsentativ für die Gesamtstichprobe ist. Die soziodemografischen Eigenschaften der verwendeten Stichprobe sind in - Tab. 1 dargestellt.

\section{Statistische Analyse der faktoriellen Struktur}

Die statistischen Analysen zur Struktur des BIP wurden mit dem Statistikprogramm R [29] und der Programmbibliothek psych [30] durchgeführt. Da uns nur die faktorielle Struktur von neu aufgetretenen Symptomen interessierte, wurden in einem ersten Schritt die Items von Abschnitt 2.3. dichotomisiert, indem die Ausprägung „neu aufgetreten“ mit 1 und die Ausprägungen „schon immer vorhanden“ und „nie vorhanden“ mit 0 kodiert wurden. Auf der Basis dieser Items wurde anschließend die Anzahl der zu extrahierenden Faktoren mittels Parallelanalyse nach Horn [31] bestimmt. Bei diesem Verfahren werden die Eigenwerte einer Faktoranalyse eines empirischen Datensatzes mit den Eigenwerten einer Faktoranalyse eines Datensatzes mit normalverteilten Zufallsdaten gleicher Größe (d.h. gleiche Anzahl Fälle und Variablen) verglichen. Der Eigenwert eines Faktors beschreibt, wie stark Items mit ihm korrelieren bzw. auf ihn laden. Die Idee der Parallelanalyse besteht darin, nur diejenigen Faktoren zu behalten, die Eigenwerte aufweisen, die größer sind als diejenigen, die auf Zufallsdaten basieren. Nachdem die Anzahl der zu extrahierenden Faktoren bestimmt war, wurde eine explorative Faktoranalyse mit ungewichteter Kleinst-QuadrateSchätzung und Oblimin-Rotation durchgeführt. Die Oblimin-Rotation ist ein schiefwinkliges Rotationsverfahren, das dazu dient, die Interpretation der gefundenen Faktorlösung zu erleichtern. Dabei werden die Faktoren so gedreht, dass jedes Item möglichst nur auf einen Faktor lädt und somit die Items eindeutig einzelnen Faktoren zugeordnet werden können. Sowohl die Parallelanalyse

\begin{tabular}{|c|c|c|c|c|c|}
\hline & Alle & Risikopersonen & Ersterkrankte & \multirow[t]{2}{*}{$p$-Wert } & \multirow{2}{*}{$\begin{array}{l}\text { Tab. } 1 \text { Soziodemografische } \\
\text { Eigenschaften. }\end{array}$} \\
\hline & $n=197$ & $n=120$ & $n=77$ & & \\
\hline Geschlecht: & & & & 0,668 & \\
\hline - Frauen (\%) & $74(37,6 \%)$ & $47(39,2 \%)$ & $27(35,1 \%)$ & & \\
\hline - Männer (\%) & $123(62,4 \%)$ & $73(60,8 \%)$ & $50(64,9 \%)$ & & \\
\hline Alter Mittelwert (SD) & $27,5(8,23)$ & $26,1(7,80)$ & $29,8(8,44)$ & $0,002 * *$ & \\
\hline Bildungsjahre Mittelwert (SD) & $11,7(3,02)$ & $11,7(3,02)$ & $11,7(3,04)$ & 0,962 & \\
\hline BPRS Gesamtscore Mittelwert (SD) & $43,6(12,5)$ & $38,5(9,07)$ & $52,6(12,7)$ & $<0,001^{* * *}$ & \\
\hline SANS Gesamtscore Mittelwert (SD) & $24,2(16,4)$ & $22,5(16,4)$ & $27,2(16,2)$ & 0,073 & \\
\hline
\end{tabular}

$\mathrm{SD}=$ Standardabweichung; BPRS = Brief Psychiatric Rating Scale, SANS = Scale for the Assessment of Negative Symptoms, ${ }^{*} \mathrm{p}<0.01$, *** $\mathrm{p}<0.001$. 
als auch die anschließende explorative Faktoranalyse beruhten auf den tetrachorischen Korrelationen zwischen den Items, da diese - im Unterschied zu den oft fälschlicherweise verwendeten Pearson-Korrelationen - nicht zu verzerrten Faktorstrukturen und -ladungen führen [vgl. 32]. Die tetrachorische Korrelation ist ein Zusammenhangsmaß von zwei dichotomen Variablen. Sie quantifiziert die Korrelation der latenten Variablen, die dichotomen Items zugrunde liegen. Um möglichst alle erhobenen Daten zu nutzen, wurden die Korrelationen jeweils mit paarweise kompletten Fällen berechnet.

Anhand der gefundenen Faktorlösung wurden neue BIP-Skalen gebildet. Die Zuordnung der Items zu den Skalen erfolgte nach ihrer Hauptladung, wobei Items mit Hauptladungen $<0,4$ entfernt wurden. Die neuen Skalen wurden auf ihre interne Konsistenz mittels Cronbach's $\alpha$ geprüft. Da dieser Koeffizient jedoch kein Beleg für die Eindimensionalität bzw. Homogenität einer Skala ist [33], wurde zusätzlich Revelle's $\beta$ [34] berechnet.

\section{Statistische Analyse der Validität}

Für die Bestimmung der Konstruktvalidität wurden die neu gebildeten BIP-Skalen mit anderen Fremdbeurteilungsskalen von verwandtem Gültigkeitsanspruch korreliert, die ebenfalls im Rahmen der FePsy-Studie bei Einschluss in die Studie erhoben worden waren. Konkret wurden dafür die Brief Psychiatric Rating Scale Expanded Version (BPRS-E) [35] und die Scale for the Assessment of Negative Symptoms (SANS) [36] verwendet. Die BPRS-Skalen wurden gemäß Velligan et al. [35] berechnet.

Um die prädiktive Validität der neu konstruierten BIP-Skalen zu testen, wurde mittels univariater Cox-Regressionsmodelle geprüft, ob die BIP-Skalen die Zeit bis zur Transition zu einer Psychose signifikant vorhersagen. Die Transition zu einer Psychose wurde in regelmäßigen Abständen anhand des BPRS und der Kriterien von Yung et al. [37] überprüft, d.h. im ersten Jahr monatlich, im zweiten und dritten Jahr dreimonatlich und danach jährlich bis maximal fünf Jahre nach Einschluss.

\section{Resultate}

$\nabla$

\section{Interrater-Reliabilität}

Von 153 untersuchten BIP-Items wiesen 124 (81\%) eine beinahe vollkommene, 23 (15\%) eine starke, 3 (2\%) eine mittelmäßige, $2(1,3 \%)$ eine leichte und $1(0,7 \%)$ eine schwache Übereinstimmung auf. Bei 151 (98,7\%) der untersuchten Items war der Interrater-Reliabilitätskoeffizient signifikant größer als 0 . Die drei einzigen Items mit ungenügender Übereinstimmung (Koeffizient $<0,4)$ betrafen die drei frühesten Auffälligkeiten im Abschnitt 2.2.2, wobei die zeitlich erste Auffälligkeit eine Interrater-Reliabilität von 0,39 , die zweite 0,23 , und die dritte 0,12 aufwies. Die drei Items mit mittelmäßiger Übereinstimmung betrafen selbstwahrgenommene Veränderungen als Grund der jetzigen Vorstellung in der Früherkennungssprechstunde (Gwet's AC1 =0,48), die Einschätzung des Zuweisungswegs (Gwet's $A C 1=0,51$ ) und die Zeitdauer seit dem ersten Auftreten des ersten Symptoms aus dem Symptombereich „Übernatürliches“ $(\operatorname{ICC}(2,1)=0,56)$. Die Interrater-Reliabilitäten der Beurteilungen von Art, Zeitdauer seit dem ersten Auftreten, Verlauf und jetziger Ausprägung des ersten Symptoms jedes Symptombereichs des Abschnitts 2.3 sind in - Tab. 2 dargestellt.

\section{Faktorielle Struktur}

Die Parallelanalyse nach Horn [31] ergab, dass die gemeinsame Varianz der BIP-Items des Abschnitts 2.3 optimal durch fünf Faktoren erklärt wird. Eine explorative Faktoranalyse mit Oblimin-Rotation und fünf Faktoren erhärtete diesen Befund, da diese Faktorlösung leicht interpretierbar war (siehe Ladungsmatrix in $\bullet$ Tab.3).

56 der 62 Items konnten einem der fünf Faktoren zugeordnet werden (Hauptladung $\geq 0,4$ ). Auf den ersten Faktor luden hauptsächlich Items, die Wahn, Halluzinationen, Ich-Störungen, Misstrauen und Beschäftigung mit Übernatürlichem erfassten, weshalb dieser Faktor den Namen „Positive psychotische Symptome“ erhielt. Der zweite Faktor erhielt den Namen „Störungen des Denkens“, da hauptsächlich Konzentrations- und Denkschwierigkeiten auf diesen Faktor luden. Der dritte Faktor wurde „Vermindertes soziales Funktionieren“ genannt, da Items, die Schwierigkeiten in der Erfüllung von Erwartungen in Familie und Schule/Beruf, Interessenverlust und sozialen Rückzug beschrieben, am stärksten auf diesen Faktor luden. Der vierte Faktor bestand vorwiegend aus Items, die

\begin{tabular}{|c|c|c|c|c|}
\hline Symptombereich & $\begin{array}{l}\text { Welches trat } \\
\text { zuerst auf? } \\
\text { Gwet's AC1 }\end{array}$ & $\begin{array}{l}\text { Zeitpunkt des } \\
\text { ersten Auftretens } \\
\text { ICC }(2,1)\end{array}$ & $\begin{array}{l}\text { Verlauf } \\
\text { Gwet's AC1 }\end{array}$ & $\begin{array}{l}\text { Ausprägung jetzt } \\
\text { Gwet's AC1 }\end{array}$ \\
\hline Sorgen & $0,836[0,640-1,000]$ & $0,856[0,413-0,863]$ & $0,816[0,597-1,000]$ & $0,819[0,579-1,000]$ \\
\hline Körperliche Beschwerden & $0,774[0,564-0,984]$ & $0,990[0,806-0,970]$ & $0,714[0,440-0,988]$ & $0,609[0,324-0,894]$ \\
\hline Denken/Konzentration & $0,938[0,833-1,000]$ & $0,958[0,916-0,982]$ & $0,845[0,688-1,000]$ & $0,937[0,829-1,000]$ \\
\hline Zwänge & $0,832[0,640-1,000]$ & $0,998[0,875-0,995]$ & $0,862[0,731-0,993]$ & $0,867[0,733-1,000]$ \\
\hline Stimmung & $0,860[0,710-1,000]$ & $0,951[0,897-0,975]$ & $0,717[0,436-0,997]$ & $0,744[0,426-1,000]$ \\
\hline Sensibilität & $0,893[0,679-1,000]$ & $0,978[0,859-0,978]$ & $0,799[0,598-1,000]$ & $0,828[0,643-1,000]$ \\
\hline Sozialer Rückzug & $0,865[0,679-1,000]$ & $0,923[0,705-0,949]$ & $0,741[0,410-1,000]$ & $0,732[0,335-1,000]$ \\
\hline Übernatürliches & $0,804[0,650-0,958]$ & $0,562[0,398-0,866]$ & $0,922[0,800-1,000]$ & $0,844[0,632-1,000]$ \\
\hline Derealisation & $0,788[0,578-0,999]$ & $0,897[0,760-0,948]$ & $0,739[0,386-1,000]$ & $0,815[0,461-1,000]$ \\
\hline Halluzinationen & $0,843[0,706-0,980]$ & $0,765[0,607-0,931]$ & $0,821[0,556-1,000]$ & $0,853[0,590-1,000]$ \\
\hline Wahn & $0,915[0,771-1,000]$ & $0,739[0,614-0,923]$ & $0,779[0,551-1,000]$ & $0,941[0,818-1,000]$ \\
\hline Gedanken & $0,971[0,904-1,000]$ & $0,905[0,782-0,991]$ & $0,979[0,918-1,000]$ & $0,980[0,918-1,000]$ \\
\hline Fremdbeeinflussung & $0,930[0,825-1,000]$ & $1,000[0,494-0,986]$ & $0,982[0,928-1,000]$ & $1,000[1,000-1,000]$ \\
\hline Soziale Anpassung & $0,764[0,561-0,966]$ & $0,907[0,817-0,960]$ & $0,923[0,624-1,000]$ & $0,853[0,556-1,000]$ \\
\hline
\end{tabular}

In eckigen Klammern sind die unteren und oberen Grenzen der 95\%-Konfidenzintervalle angegeben. 
Tab. 3 Ladungsmatrix der explorativen Faktoranalyse mit 5 Faktoren.

Item

F1

\section{Faktor 1: Positive psychotische Symptome}

2.3.44 Alltägliches hatte besondere Bed

2.3.32 übernatürliche Dinge erlebt

2.3.46 Will jemand Ihnen absichtlich Schaden zufügen, Sie vergiften, töten, verfolgen?

2.3.52 unter Kontrolle von Kräften/Mächten?

2.3.45 Leute, die Ihnen gegenüber viele Anspielungen, Doppeldeutigkeiten machten

2.3.31 ungewöhnliches Interesse für Religion, übernatürliche Dinge

2.3.49 fremde Gedanken in Ihrem Kopf?

2.3.48 Sie können Dinge durch magisches Denken beeinflussen?

2.3.40 Geräusche gehört, auch wenn niemand da war

2.3.42 Stimmen, die über Sie sprachen, sich miteinander unterhielten?

2.3.41 Stimmen gehört?

2.3.50 Gedanken werden laut, breiten sich aus

2.3.28 ungewöhnlich misstrauisch?

2.3.25 über das normale Maß überschwenglich oder hyperaktiv?

2.3.47 außergewöhnliche übernatürliche Fähigkeiten?

Faktor 2: Störungen des Denkens

2.3.10 ungewohnte Schwierigkeiten beim Denken

2.3.11 Gedanken durcheinander?

2.3.13 Gedanken blockiert, plötzlich gestoppt

2.3.12 schieben andere Gedanken in Gedankenfluss ein?

2.3.16 Zwang, Dinge zu ordnen, waschen, zählen, ganz unsinnig

2.3.15 Schwierigkeiten zu verstehen, was andere sprechen?

2.3.09 ungewöhnliche Schwierigkeiten, sich zu konzentrieren

2.3.51 Gedanken aus dem Kopf entzogen?

2.3.24 gereizt, verärgert, ungewöhnlich viel Streit?

2.3.14 ungewöhnlich vergesslich?

2.3.07 energielos, kraftlos, verlangsamt

2.3.22 erstarrte Gefühle?

2.3.20 absichtlich verletzt?

Faktor 3: Vermindertes soziales Funktionieren

2.3.59 Erwartungen Ihrer Familie nicht mehr erfüllt?

2.3.61 Erwartungen in Schule/Beruf nicht mehr erfüllt?

2.3.62 nicht mehr regelmäßig zur Arbeit/Schule?

2.3.55 Interesse verloren, in Ihrer Freizeit nichts unternommen?

2.3.60 Arbeit/Schule sehr viel anstrengender?

2.3.54 ungewöhnliche Vernachlässigung der Körperhygiene und Kleidung

2.3.57 von Ihrer Familie zurückgezogen?

2.3.58 aus Partnerschaft ganz zurückgezogen?

2.3.29 zurückgezogen, den Kontakt zu anderen vermieden?

2.3.53 Dinge des Alltags mit viel Mühe, sehr langsam bewältigt?

2.3.56 von Ihren Freunden zurückgezogen?

Faktor 4: Depression/Angst

2.3.17 sehr bedrückt, nicht mehr so freuen?

2.3.01 mehr Sorgen als früher, kaum abschalten können

2.3.27 ungewöhnlich empfindlich, dünnhäutig?

2.3.18 ernsthaft gedacht, sich umzubringen?

2.3.08 Nachlassen des sexuellen Interesses

2.3.26 unsicher im Umgang mit anderen Personen?

2.3.19 Selbstmordversuch begangen?

2.3.21 Schuldgefühle?

2.3.02 nervöser, mehr innere Unruhe

2.3.23 wechselhafte Gefühle, schlagartig wechselnd, von positiv in negativ?

2.3.05 Veränderung des Appetits

\section{Faktor 5: Ungewöhnliche Erfahrungen}

2.3.35 Dinge, die ungewöhnlich aussahen, sich anhörten, rochen, schmeckten?

2.3.30 ungewöhnliche Verhaltensweisen?

2.3.43 Dinge gesehen, die andere Leute nicht gesehen haben?

2.3.37 selber unwirklich zu sein

2.3.36 ungewöhnliche Körperempfindungen?

2.3.03 ungewöhnliche Ängste, Furcht

2.3.34 unwirkliche Umgebung (fremd, wie im Theater)

2.3.38 veränderte Form der Körperteile
0,75

0,72

0,64

0,62

0,62

0,55

0,54

0,53

0,50

0,48

0,48

0,45

$\mathbf{0 , 4 5}$

$\mathbf{0 , 4 4}$

0,34

$-0,04$

0,04

0,11

0,19

0,11

0,14

$-0,37$

0,40

0,13

$-0,16$

$-0,27$

0,15

0,04

0,22

$-0,11$

0,08

$-0,19$

$-0,26$

0,07

0,04

0,18

0,05

$-0,19$

0,10

$-0,03$

$-0,17$

0,22

0,23

0,02

0,18

0,02

0,23

$-0,19$

0,08

$-0,06$

0,23

0,11

0,36

$-0,07$

0,30

0,00

$-0,01$

0,36
F2

F3

F4

F5

$\begin{array}{llll}-0,01 & 0,04 & -0,04 & 0,04\end{array}$

$\begin{array}{rrrr}-0,19 & -0,09 & 0,23 & 0,18\end{array}$

$\begin{array}{llll}0,16 & -0,08 & -0,14 & 0,06\end{array}$

$\begin{array}{rrrr}0,16 & 0,04 & -0,08 & 0,16\end{array}$

$\begin{array}{llll}0,23 & -0,08 & 0,09 & -0,14\end{array}$

$\begin{array}{llll}-0,18 & 0,11 & 0,44 & 0,13\end{array}$

$\begin{array}{rrrr}0,19 & 0,10 & 0,01 & 0,15\end{array}$

$\begin{array}{llll}0,03 & -0,04 & 0,12 & 0,17\end{array}$

$\begin{array}{llll}0,16 & 0,21 & -0,17 & 0,11\end{array}$

$\begin{array}{rrrr}0,29 & 0,30 & 0,02 & 0,05\end{array}$

$\begin{array}{rrrr}0,22 & 0,12 & -0,08 & 0,13\end{array}$

$\begin{array}{llll}0,38 & 0,26 & 0,06 & -0,10\end{array}$

$\begin{array}{rrrr}0,12 & 0,18 & 0,07 & 0,04\end{array}$

$\begin{array}{rrrr}0,35 & -0,02 & 0,17 & -0,27\end{array}$

$\begin{array}{llll}-0,12 & 0,03 & 0,32 & 0,30\end{array}$

$\mathbf{0 , 7 9} \quad-0,08 \quad 0,06 \quad 0,01$

$\begin{array}{rrrr}\mathbf{0 , 6 8} & -0,07 & -0,01 & 0,25\end{array}$

$\begin{array}{llll}\mathbf{0 , 6 8} & 0,05 & 0,05 & -0,04\end{array}$

$\begin{array}{llll}\mathbf{0 , 6 7} & 0,02 & -0,15 & 0,11\end{array}$

$\begin{array}{llll}\mathbf{0 , 6 0} & -0,16 & -0,03 & 0,20\end{array}$

$\begin{array}{llll}\mathbf{0 , 5 9} & 0,26 & 0,03 & -0,11\end{array}$

$\mathbf{0 , 5 5} \quad 0,15 \quad 0,14 \quad 0,11$

$\begin{array}{llll}\mathbf{0 , 5 0} & 0,23 & -0,09 & 0,08\end{array}$

$\begin{array}{llll}\mathbf{0 , 4 9} & -0,09 & 0,15 & -0,09\end{array}$

$\begin{array}{llll}\mathbf{0 , 4 9} & 0,25 & 0,09 & 0,15\end{array}$

$\begin{array}{rrrr}\mathbf{0 , 4 2} & 0,29 & 0,24 & 0,08\end{array}$

$\begin{array}{llll}\mathbf{0 , 4 1} & 0,31 & 0,24 & -0,04\end{array}$

$\begin{array}{llll}0,13 & 0,12 & 0,10 & 0,07\end{array}$

$\begin{array}{llll}-0,12 & \mathbf{0 , 7 8} & -0,04 & -0,06\end{array}$

$\begin{array}{llll}-0,08 & \mathbf{0 , 7 7} & -0,23 & 0,14\end{array}$

$\begin{array}{llll}-0,15 & \mathbf{0 , 7 3} & -0,05 & -0,07\end{array}$

$\begin{array}{llll}0,19 & \mathbf{0 , 6 0} & 0,26 & -0,09\end{array}$

$\begin{array}{rrrr}0,18 & \mathbf{0 , 5 9} & 0,06 & 0,26\end{array}$

$\begin{array}{llll}0,15 & \mathbf{0 , 5 6} & 0,17 & -0,19\end{array}$

$\begin{array}{rrrr}-0,03 & \mathbf{0 , 5 4} & 0,34 & 0,08\end{array}$

$\begin{array}{rrrr}0,13 & \mathbf{0 , 5 1} & -0,03 & 0,12\end{array}$

$\begin{array}{llll}0,08 & \mathbf{0 , 4 8} & 0,36 & -0,07\end{array}$

$\begin{array}{rrrr}0,19 & \mathbf{0 , 4 8} & 0,22 & 0,16\end{array}$

$\begin{array}{llll}0,24 & 0,39 & 0,32 & -0,13\end{array}$

$\begin{array}{rrrr}0,09 & 0,17 & \mathbf{0 , 6 9} & -0,01\end{array}$

$\begin{array}{rrrr}-0,19 & -0,16 & \mathbf{0 , 6 8} & 0,30\end{array}$

$\begin{array}{llll}0,23 & -0,08 & \mathbf{0 , 5 3} & -0,08\end{array}$

$\begin{array}{rrrr}-0,07 & 0,14 & \mathbf{0 , 5 3} & -0,18\end{array}$

$\begin{array}{llll}0,18 & 0,04 & \mathbf{0 , 5 0} & 0,06\end{array}$

$\begin{array}{llll}0,19 & 0,07 & \mathbf{0 , 4 7} & -0,28\end{array}$

$\begin{array}{llll}0,09 & 0,08 & \mathbf{0 , 4 6} & -0,12\end{array}$

$\begin{array}{rrrr}-0,04 & 0,24 & \mathbf{0 , 4 6} & 0,09\end{array}$

$\begin{array}{rrrr}0,29 & -0,08 & \mathbf{0 , 4 3} & 0,20\end{array}$

$\begin{array}{llll}0,37 & -0,23 & \mathbf{0 , 4 1} & -0,19\end{array}$

$\begin{array}{llll}0,12 & 0,20 & 0,28 & 0,21\end{array}$

$\begin{array}{llll}0,10 & 0,07 & -0,02 & \mathbf{0 , 6 6}\end{array}$

$\begin{array}{llll}0,15 & 0,02 & -0,02 & \mathbf{0 , 5 2}\end{array}$

$\begin{array}{llll}0,04 & 0,17 & 0,05 & \mathbf{0 , 5 0}\end{array}$

$\begin{array}{llll}0,26 & -0,06 & -0,03 & \mathbf{0 , 5 0}\end{array}$

$\begin{array}{llll}-0,12 & -0,02 & 0,05 & \mathbf{0 , 4 9}\end{array}$

$\begin{array}{llll}0,03 & -0,12 & 0,42 & \mathbf{0 , 4 9}\end{array}$

$\begin{array}{rrrr}0,25 & 0,12 & 0,13 & \mathbf{0 , 4 5}\end{array}$

$\begin{array}{llll}-0,08 & 0,16 & -0,19 & \mathbf{0 , 4 4}\end{array}$ 


\begin{tabular}{|c|c|c|c|c|c|c|}
\hline Nr. & Item & F1 & F2 & F3 & F4 & F5 \\
\hline 2.3 .04 & ungewöhnlich viele Kopfschmerzen, andere körperliche Beschwerden & $-0,01$ & 0,24 & 0,00 & $-0,09$ & 0,43 \\
\hline 2.3 .33 & Gefühl, es gehen unerklärliche Dinge vor sich & 0,35 & 0,20 & $-0,05$ & 0,10 & 0,40 \\
\hline 2.3 .39 & Schwierigkeiten, Vorstellungen von echten Wahrnehmungen zu unterscheiden & 0,20 & 0,32 & $-0,12$ & 0,11 & 0,39 \\
\hline 2.3.06 & Schlafstörung über mehr als 1 Woche & $-0,13$ & $-0,03$ & 0,15 & 0,20 & 0,34 \\
\hline
\end{tabular}

Die Items sind nach der absoluten Höhe ihrer Hauptladung in absteigender Reihenfolge sortiert. Items mit fett markierten Ladungen wurden den entsprechenden Faktoren zugeordnet.

\begin{tabular}{|c|c|c|c|c|c|c|c|}
\hline \multirow[t]{2}{*}{ BIP-Skalen } & \multirow[t]{2}{*}{$\mathrm{N}$ items } & \multicolumn{2}{|l|}{ Alle } & \multicolumn{2}{|c|}{ Risikopatienten } & \multicolumn{2}{|c|}{ Ersterkrankte } \\
\hline & & alpha $^{1}$ & beta $^{2}$ & alpha $^{1}$ & beta $^{2}$ & alpha ${ }^{1}$ & beta $^{2}$ \\
\hline Positive psychotische Symptome & 14 & 0,90 & 0,84 & 0,81 & 0,72 & 0,87 & 0,58 \\
\hline Störungen des Denkens & 12 & 0,89 & 0,70 & 0,84 & 0,70 & 0,88 & 0,57 \\
\hline Vermindertes soziales Funktionieren & 10 & 0,86 & 0,79 & 0,80 & 0,66 & 0,88 & 0,79 \\
\hline Depression/Angst & 10 & 0,80 & 0,63 & 0,80 & 0,59 & 0,73 & 0,41 \\
\hline Ungewöhnliche Erfahrungen & 10 & 0,82 & 0,73 & 0,81 & 0,43 & 0,72 & 0,41 \\
\hline Gesamtscore & 62 & 0,95 & 0,51 & 0,91 & 0,60 & 0,94 & 0,14 \\
\hline
\end{tabular}

affektive Symptome erfassen, und wurde deshalb „Depression/ Angst“ genannt. Der fünfte Faktor erhielt schließlich den Namen „Ungewöhnliche Erfahrungen“, da Items, die ungewöhnliche Wahrnehmungen, Empfindungen, Gefühle, Verhaltensweisen bezeichneten, am stärksten auf diesen Faktor luden.

Die Ergebnisse der Reliabilitätsanalyse der fünf neu gebildeten Skalen sowie der Gesamtskala sind in $\bullet$ Tab. 4 dargestellt. Die internen Konsistenzen waren für alle Skalen sowohl in der Gesamtstichprobe als auch in der Gruppe der Ersterkrankten und Risikopatienten ausreichend hoch $(\alpha>0,7)$ und größtenteils sogar im guten bis sehr guten Bereich ( $\alpha>0,8)$. Die Homogenitäten (Revelle’s $\beta$ ) waren überwiegend $>0,5$, was gemäß Revelle [38] einen ausreichend hohen Wert darstellt. Unbefriedigende Homogenitäten $(\beta<0,5)$ traten lediglich in der Gesamtskala und in den Skalen „Depression/Angst“ und „Störungen des Denkens“ in der Gruppe der Ersterkrankten auf.

\section{Validität}

Die Korrelationen der BIP-Skalen mit den Skalen des BPRS-E und der SANS sind in $\bullet$ Tab. 5 dargestellt. BIP „Positive psychotische Symptome“ korrelierte signifikant mit BPRS „Positive Symptome“ $(p<0,001)$ und BPRS Gesamtscore $(p<0,001)$, BIP „Störungen des Denkens“ korrelierte signifikant mit BPRS „Positive Symptome“ $(p=0,022)$, BIP „Vermindertes soziales Funktionieren“ korrelierte signifikant mit SANS „Apathie/Antriebsarmut“ $(\mathrm{p}<0,001)$ und SANS „Anhedonie/Disssozialität“ $(p=0,016)$, SANS Globalscore $(p=0,025)$ und SANS Gesamtscore $(p=0,006)$, BIP „Depression/ Angst“ korrelierte signifikant mit BPRS „Depression/Angst“ ( $p<0,001)$, und BIP „Ungewöhnliche Erfahrungen“ korrelierte signifikant mit BPRS „Depression/Angst“ $(p=0,012)$, BPRS „Positive Symptome“ ( $p<0,001)$ und BPRS Gesamtscore $(p=0,005)$. Die hohen Korrelationen mit Skalen von verwandtem Gültigkeitsanspruch und die niedrigen Korrelationen mit Skalen von nicht verwandtem Gültigkeitsanspruch wiesen auf eine gute konvergente und divergente Validität aller fünf BIP-Skalen hin. Die Ergebnisse der Cox-Regressionsanalysen sind in $\bullet$ Tab. 6 dargestellt. Von den 120 untersuchten ARMS-Patienten hatten 31 im
Verlauf der Follow-ups eine Psychose entwickelt. Die durchschnittliche Dauer bis zur Transition betrug 394 Tage ( $S D=403$, Minimum =11, Maximum =1664, Median =262). Drei der sechs BIP Skalen waren signifikante Prädiktoren für eine spätere psychotische Dekompensation: „Positive psychotische Symptome“ $(p=0,007)$, „Störungen des Denkens“ $(p=0,029)$ und der BIP Gesamtscore $(p=0,017)$. Hohe Werte in jeder dieser Skalen erhöhten das Risiko für die Entwicklung einer Psychose.

\section{Diskussion}

In der vorliegenden Studie wurden zum ersten Mal die psychometrischen Eigenschaften des BIP untersucht. Wir konnten nachweisen, dass sich das BIP hervorragend eignet, um frühe Anzeichen und Symptome sowie die Vulnerabilität von Psychoserisikopersonen und Psychoseersterkrankten zu erfassen.

\section{Interrater-Reliabilität}

Von 153 untersuchten Items der Abschnitte 2 und 3 wurden 150 (98\%) mit ausreichend hoher Übereinstimmung (Interrater-Reliabilität >0,4) eingeschätzt. Bei 81 \% der Items war die Übereinstimmung sogar beinahe vollkommen (Interrater-Reliabilität $>0,8)$. Dies deutet darauf hin, dass frühe Anzeichen und Symptome - auch wenn diese zum Teil Jahre zurückliegen - von geschultem Personal mit hoher Übereinstimmung durch das BIP eingeschätzt werden können.

Die drei einzigen Items mit ungenügender Übereinstimmung betrafen die Beurteilungen der frühesten psychopathologischen Auffälligkeiten, die der Patient selbst bemerkte (Abschnitt 2.2.2). Gemäß Instruktion ist der Rater bei diesen Items aufgefordert, zunächst die berichteten Veränderungen in zeitlicher Reihenfolge ihres Auftretens und in den Worten des Patienten zu notieren und danach entsprechend den in Abschnitt 2.3 beschriebenen Symptomen zu kodieren. Da Letzteres erst nach dem Interview gemacht werden muss, wurde es von einigen Ratern vergessen. Dies wiederum könnte dazu geführt haben, dass die Überein- 
Tab. 5 Korrelationen zwischen BIP-, BPRS- und SANS-Skalen.

\begin{tabular}{|c|c|c|c|c|c|}
\hline & $\begin{array}{l}\text { Positive } \\
\text { psychotische } \\
\text { Symptome }\end{array}$ & $\begin{array}{l}\text { Störungen } \\
\text { des Denkens }\end{array}$ & $\begin{array}{l}\text { Vermindertes } \\
\text { soziales } \\
\text { Funktionieren }\end{array}$ & $\begin{array}{l}\text { Depression/ } \\
\text { Angst }\end{array}$ & $\begin{array}{l}\text { Ungewöhnliche } \\
\text { Erfahrungen }\end{array}$ \\
\hline BPRS Depression/Angst & 0,17 & 0,18 & 0,13 & $0,27^{*}$ & $0,29 * *$ \\
\hline BPRS Positive Symptome & $0,49^{* * *}$ & $0,26^{*}$ & 0,23 & 0,15 & $0,36^{* * *}$ \\
\hline BPRS Negative Symptome & 0,05 & 0,08 & 0,24 & 0,11 & 0,06 \\
\hline BPRS Aktivierung & 0,20 & 0,04 & 0,07 & 0,06 & 0,17 \\
\hline BPRS total & $0,36^{* * *}$ & 0,22 & 0,21 & 0,18 & $0,29 * *$ \\
\hline SANS Affektive Abflachung & $-0,02$ & 0,09 & 0,20 & 0,10 & 0,04 \\
\hline SANS Alogie & $-0,02$ & 0,15 & 0,12 & 0,02 & $-0,04$ \\
\hline SANS Apathie/Antriebsarmut & 0,18 & 0,22 & $0,35^{* * *}$ & 0,14 & 0,05 \\
\hline SANS Anhedonie/Dissozialität & 0,15 & 0,14 & $0,28^{*}$ & 0,21 & 0,01 \\
\hline SANS Aufmerksamkeit & 0,16 & 0,17 & 0,21 & 0,11 & $-0,05$ \\
\hline SANS global & 0,11 & 0,19 & $0,26^{*}$ & 0,14 & 0,01 \\
\hline SANS total & 0,09 & 0,17 & $0,29 * *$ & 0,15 & 0,01 \\
\hline
\end{tabular}

BIP = Basler Interview für Psychosen; BPRS = Brief Psychiatric Rating Scale; SANS = Scale for the Assessment of Negative Symptoms; ${ }^{*} p<0.05,{ }^{* *} p<0.01,{ }^{* * *} p<0.001$

\begin{tabular}{|c|c|c|c|c|c|}
\hline & Keine Transition & Transition & \multirow[t]{2}{*}{ HR [95\%-KI] } & \multirow[t]{2}{*}{$p$-Wert } & \multirow{8}{*}{$\begin{array}{l}\text { Tab. } 6 \text { Prädiktive Validität der } \\
\text { neuen BIP-Skalen. }\end{array}$} \\
\hline & $n=89$ & $n=31$ & & & \\
\hline Positive psychotische Symptome & $2,00(2,08)$ & $3,19(2,39)$ & $1,18[1,05 ; 1,33]$ & $0,007^{* *}$ & \\
\hline Störungen des Denkens & $5,21(2,67)$ & $6,32(2,52)$ & $1,16[1,02 ; 1,33]$ & $0,029^{*}$ & \\
\hline Vermindertes soziales Funktionieren & $5,21(2,40)$ & $5,84(2,96)$ & $1,10[0,95 ; 1,27]$ & 0,212 & \\
\hline Depression Angst & $5,61(2,33)$ & $6,35(2,14)$ & $1,12[0,96 ; 1,31]$ & 0,149 & \\
\hline Ungewöhnliche Erfahrungen & $2,72(2,19)$ & $2,84(2,28)$ & $1,07[0,92 ; 1,24]$ & 0,394 & \\
\hline Gesamtscore & $22,9(8,21)$ & $26,8(8,89)$ & $1,05[1,01 ; 1,10]$ & $0,017^{*}$ & \\
\hline
\end{tabular}

$\mathrm{HR}=$ Hazard Ratio; $\mathrm{KI}=$ Konfidenzintervall, ${ }^{*} \mathrm{p}<0.05,{ }^{* *} \mathrm{p}<0.01$.

stimmung relativ niedrig ausgefallen ist. Ein anderer möglicher Grund ist, dass beim Rater-Training die korrekte Bewertung dieser Items zu wenig geübt wurde. Um die Interrater-Reliabilität dieser Items in zukünftigen Erhebungen zu verbessern, haben wir deshalb am Ende des Abschnitts 2.3 einen Hinweis zur Codierung der Items 2.2.2 neu eingefügt. Zusätzlich weisen wir neu im Manual darauf hin, dass die korrekte Beurteilung der Items 2.2.2 eher anspruchsvoll sei und deshalb beim Rater-Training besonders intensiv geübt werden müsse.

\section{Faktorielle Struktur}

Eine Untersuchung der Faktorstruktur der Items des Abschnitts 2.3 in einer Stichprobe von 77 Psychoserisikopersonen und 120 Psychoseersterkrankten ergab, dass eine fünf-faktorielle Lösung am besten zu den Daten passte. Auf der Basis der gefundenen Faktorlösung wurden fünf neue Skalen konstruiert, in deren Berechnung 56 der 62 analysierten Items eingingen. Die neuen Skalen wurden wie folgt benannt: 1. Positive psychotische Symptome, 2. Störungen des Denkens, 3. Vermindertes soziales Funktionieren, 4. Depression/Angst und 5. Ungewöhnliche Erfahrungen. Zudem wurde der Gesamtscore auf der Basis aller 62 Items berechnet. Es zeigte sich, dass die neuen Skalen in den untersuchten Stichproben überwiegend gute bis sehr gute interne Konsistenzen (Cronbach's $\alpha$ ) und Homogenitäten (Revelle's $\beta$ ) aufwiesen. Korrelationsanalysen mit Skalen von verwandtem und nicht-verwandtem Gültigkeitsanspruch wiesen zudem auf gute konvergente und diskriminante Validitäten der BIP-Skalen hin.

Die gefundene faktorielle Struktur bestätigt in einigen Aspekten die Resultate früherer Studien zur Dimensionalität psychopathologischer Symptome bei ARMS- und FEP-Patienten. So weist die faktorielle Struktur des BIP zum Beispiel große Überschneidungen mit den 8 Psychose-Dimensionen des Diagnostic and Statistical Manual of Mental Disorders, Version 5, (DSM-5) [39] auf. Auch im DSM-5 gibt es eine eigenständige Dimension, die kognitive Beeinträchtigungen abdeckt. Weiter weisen die DSM5-Dimensionen „Depression“ und „Negative Symptome“ große Ähnlichkeiten mit den BIP-Faktoren „Depression/Angst“ und „Vermindertes soziales Funktionieren“ auf. Während jedoch im DSM-5 Halluzinationen und Wahn in getrennten Faktoren abgebildet werden, sind sie im BIP in einem einzigen Faktor „Positive psychotische Symptome“ vereint. Die Frage, ob Wahn und Halluzinationen eine einzige oder zwei gesonderte Dimensionen darstellen, wurde jedoch auch schon im Vorfeld der Veröffentlichung des DSM-5 kontrovers diskutiert [vgl. 40]. Auch bei dimensionalen Analysen der Scale of Prodromal Symptoms (SOPS) und der CAARMS [41-44] gruppierten sich die Items oft sehr ähnlich wie in dieser Studie. Ein genauer Vergleich der faktoriellen Struktur ist jedoch schwierig, da SOPS und CAARMS nur Symptome erfassen, die aktuell vorhanden sind, während beim BIP alle Symptome beurteilt werden müssen, die seit dem 12. Lebensjahr neu aufgetreten sind. Zudem basierte die vorliegende faktorielle Analyse auf einem gemischten Sample von Psychoserisiko- und Psychoseersterkrankten, während die dimensionalen Analysen der SOPS und der CAARMS auf Stichproben von Psychoserisikopatienten beruhten. Eine weitere Einschränkung ist, dass sich die Itempools von BIP, SOPS und CAARMS nur teilweise überlappen.

\section{Validität}

Für drei der sechs BIP-Skalen („Positive psychotische Symptome“, „Störungen des Denkens“ und Gesamtscore) konnte in dieser Stu- 
die auch eine prädiktive Validität nachgewiesen werden, da hohe Werte auf diesen Skalen mit einem höheren Risiko für eine spätere Psychoseentwicklung einhergingen. Die prädiktive Validität der BIP-Skala „Positive psychotische Symptome“ stimmt mit einer Vielzahl von Studien überein, die ebenfalls einen prädiktiven Wert von positiven psychotischen Symptomen nachweisen konnten (z. B. [10, 45 - 47]). Die Tatsache, dass diese Skala in den Cox-Regressionsmodellen die höchste Signifikanz aufwies, unterstreicht die Bedeutung der genauen und ausführlichen Erfassung von abgeschwächten positiv-psychotischen Symptomen bei Psychoserisikopersonen.

Die prädiktive Validität der BIP-Skala „Störungen des Denkens“ bestätigt ebenfalls zahlreiche frühere Studien, die gezeigt haben, dass sowohl selbstberichtete [48] als auch objektiv gemessene kognitive Beeinträchtigungen $[10,49,50]$ maßgeblich zur Prädiktion von Psychosen beitragen. Zudem stimmt dieser Befund mit zwei Faktoranalysen des SOPS [42] und CAARMS [43] überein. In beiden Studien erwiesen sich Faktoren, die Items zu kognitiven Beeinträchtigungen enthielten, als prädiktiv für eine spätere Psychoseentwicklung. In der Studie von Demjaha et al. [43] war dies der SOPS-Faktor „Disorganization/cognitive“ und in der Studie von Raballo et al. [43] der CAARMS-Faktor „Disorganized“.

\section{Take Home Message}

Mit dem Basler Interview für Psychosen (BIP) steht erstmals ein halbstrukturiertes klinisches Anamneseinterview zur Verfügung, das sowohl in der Forschung als auch in der klinischen Routine verwendet werden kann, um Psychoserisikopatienten und Psychoseersterkrankte detailliert über Psychoserisikofaktoren und -indikatoren sowie den psychopathologischen Verlauf seit der Kindheit zu befragen. Nach einem vergleichsweise kurzen Rater-Training kann das Instrument von klinisch erfahrenen Fachpersonen verwendet werden.

Interessenkonflikt: Die Autoren geben an, dass kein Interessenkonflikt besteht.

\section{Literatur}

1 Daneault JG, Stip E, Refer OSG. Genealogy of instruments for prodrome evaluation of psychosis. Front Psychiatry 2013; 4: 25

2 Huber G, Gross G. The concept of basic symptoms in schizophrenic and schizoaffective psychoses. Recenti Prog Med 1989; 80: 646-652

3 Klosterkötter J, Hellmich M, Steinmeyer EM et al. Diagnosing schizophrenia in the initial prodromal phase. Arch Gen Psychiatry 2001; 58: $158-164$

4 Fusar-Poli P, Borgwardt S, Bechdolf $A$ et al. The psychosis high-risk state: a comprehensive state-of-the-art review. JAMA Psychiatry 2013; 70: $107-120$

5 Schultze-Lutter F, Addington J, Ruhrmann S et al. Schizophrenia proneness instrument, adult version (SPI-A). Rome: Giovanni Fioriti Editore srl; 2007

6 Miller TJ, McGlashan TH, Rosen JL et al. Prodromal assessment with the structured interview for prodromal syndromes and the scale of prodromal symptoms: predictive validity, interrater reliability, and training to reliability. Schizophr Bull 2003; 29: 703-715

7 Yung AR, Yuen HP, McGorry PD et al. Mapping the onset of psychosis: the Comprehensive Assessment of At-Risk Mental States. Aust N Z J Psychiatry 2005; 39: $964-971$

8 Riecher-Rössler A, Aston J, Borgwardt S et al. Prediction of psychosis by stepwise multilevel assessment-the Basel FePsy (early recognition of psychosis)-Project. Fortschr Neurol Psychiatr 2013; 81: 265-275
9 Riecher-Rössler A, Gschwandtner U, Aston J et al. The Basel early-detection-of-psychosis (FEPSY)-study-design and preliminary results. Acta Psychiatr Scand 2007; 115: 114-125

10 Riecher-Rössler A, Pflueger MO, Aston J et al. Efficacy of using cognitive status in predicting psychosis: a 7-year follow-up. Biol Psychiatry 2009; 66: $1023-1030$

11 Riecher-Rössler A, Aston J, Ventura J et al. The Basel Screening Instrument for Psychosis (BSIP): development, structure, reliability and validity. Fortschr Neurol Psychiatr 2008; 76: 207-216

12 Häfner H, Riecher-Rössler A, Hambrecht $M$ et al. IRAOS: an instrument for the assessment of onset and early course of schizophrenia. Schizophr Res 1992; 6: 209-223

13 Häfner H, Maurer K, Loffler W et al. The ABC Schizophrenia Study: a preliminary overview of the results. Soc Psychiatry Psychiatr Epidemiol 1998; 33: 380 - 386

14 Aston J, Bull N, Gschwandtner $U$ et al. First self-perceived signs and symptoms in emerging psychosis compared with depression. Early Interv Psychiatry 2012; 6: 455-459

15 Bugra H, Studerus E, Rapp C et al. Cannabis use and cognitive functions in at-risk mental state and first episode psychosis. Psychopharmacology 2013; 230: 299-308

16 Rapp C, Walter A, Studerus E et al. Cannabis use and brain structural alterations of the cingulate cortex in early psychosis. Psychiatry Res 2013; 214: $102-108$

17 Fridgen GJ, Aston J, Gschwandtner $U$ et al. Help-seeking and pathways to care in the early stages of psychosis. Soc Psychiatry Psychiatr Epidemiol 2013; 48: 1033-1043

18 Rapp C, Studerus E, Bugra H et al. Duration of untreated psychosis and cognitive functioning. Schizophr Res 2013; 145: 43-49

19 Weiss M. Explanatory Model Interview Catalogue (EMIC): Framework for comparatory study of illness. Transcult Psychiatry 1997; 34: $235-$ 263

20 Thurm-Mussgay I, Galle K, Häfner H. Krankheitsbewältigung Schizophrener: Ein theoretisches Konzept zu ihrer Erfassung und erste Erfahrungen mit einem neuen Messinstrument. Verhaltenstherapie 1991; 1: $293-300$

21 Gross G, Huber G, Klosterkötter J et al. Bonner Skala für die Beurteilung von Basissymptomen. Berlin: Springer Verlag1987

22 Miller TJ, McGlashan TH, Rosen JL et al. Prodromal assessment with the structured interview for prodromal syndromes and the scale of prodromal symptoms: predictive validity, interrater reliability, and training to reliability. Schizophr Bull 2003; 29: 703-715

23 Gwet KL. AgreeStat 2011.3 for Windows and Excel [Computer software]. Im Internet: http://www.agreestat.com/; 2011

24 Gwet KL. Computing inter-rater reliability and its variance in the presence of high agreement. Br J Math Stat Psychol 2008; 61: 29-48

25 Wongpakaran $N$, Wongpakaran $T$, Wedding $D$ et al. A comparison of Cohen's Kappa and Gwet's AC1 when calculating inter-rater reliability coefficients: a study conducted with personality disorder samples. BMC Med Res Methodol 2013: 13

26 Gwet KL. Handbook of inter-rater reliability: The definitive duide to measuring the extent of agreement among multiple raters.. 3. Aufl. Gaithersburg, MD: Advanced Analytics, LLC; 2012

27 Shrout PE, Fleiss JL. Intraclass correlations: uses in assessing rater reliability. Psychol Bull 1979; 86: 420-428

28 Landis JR, Koch GG. A One-Way Components of variance model for categorical data. Biometrics 1977; 33: 671-679

29 R Core Team. R: A language and environment for statistical computing (Version 3.0.3) [Computer software]. Im Internet: http://www.Rpro ject.org.; 2014

30 Revelle W. psych: Procedures for Psychological, Psychometric, and Personality Research (Version 1.4.5) [Computer software]. Im Internet: http://personality-project.org/r/src/contrib/; 2014

31 Horn JL. A rationale and test for the number of factors in factor analysis. Psychometrika 1965; 30: 179-185

32 Kubinger $K D$. On artificial results due to using factor analysis for dichotomous variables. Psychol Sci 2003; 45: 106-110

33 Revelle W, Zinbarg RE. Coefficients alpha beta, omega and the glb: Comments on Sijtsma. Psychometrika 2009; 74: 145-154

34 Zinbarg RE, Revelle W, Yovel I et al. Cronbach's alpha, Revelle's beta, and McDonald's (omega H): Their relations with each other and two alternative conceptualizations of reliability. Psychometrika 2005; 70: 123 - 133

35 Velligan D, Prihoda T, Dennehy E et al. Brief Psychiatric Rating Scale Expanded Version: How do new items affect factor structure? Psychiatry Res 2005; 135: 217-228 
36 Andreasen NC. The Scale for the Assessment of Negative Symptoms (SANS): conceptual and theoretical foundations. Br J Psychiatry Suppl 1989; Nov: 49-58

37 Yung AR, Phillips LJ, McGorry PD et al. Prediction of psychosis. A step towards indicated prevention of schizophrenia. Br J Psychiatry Suppl 1998; 172: $14-20$

38 Revelle W. Hierarchical Cluster-analysis and the internal structure of tests. Multivar Behav Res 1979; 14: $57-74$

39 American Psychiatric Association. Diagnostic and statistical manual of mental disorders DSM-5. 5. Aufl. Arlington, VA.: American Psychiatric Association; 2013

40 Barch DM, Bustillo J, Gaebel W et al. Logic and justification for dimensional assessment of symptoms and related clinical phenomena in psychosis: relevance to DSM-5. Schizophr Res 2013; 150: 15-20

41 Hawkins KA, McGlashan TH, Quinlan D et al. Factorial structure of the Scale of Prodromal Symptoms. Schizophr Res 2004; 68: 339-347

42 Demjaha A, Valmaggia L, Stahl D et al. Disorganization cognitive and negative symptom dimensions in the at-risk mental state predict subsequent transition to psychosis. Schizophr Bull 2012; 38: 351 - 359

43 Raballo A, Nelson B, Thompson A et al. The comprehensive assessment of at-risk mental states: from mapping the onset to mapping the structure. Schizophr Res 2011; 127: 107-114
44 Lemos S, Vallina O, Fernandez P et al. Predictive validity of the Scale of Prodromal Symptoms (SOPS). Actas Esp Psiquiatr 2006; 34: 216-223

45 Nelson B, Yuen HP, Wood SJ et al. Long-term follow-up of a group at ultra high risk ("prodromal") for psychosis: the PACE 400 study. JAMA Psychiatry 2013; 70: 793-802

46 Ruhrmann S, Schultze-Lutter F, Salokangas RK et al. Prediction of psychosis in adolescents and young adults at high risk: results from the prospective European prediction of psychosis study. Arch Gen Psychiatry 2010; $67: 241-251$

47 Cannon TD, Cadenhead K, Cornblatt B et al. Prediction of psychosis in youth at high clinical risk: a multisite longitudinal study in North America. Arch Gen Psychiatry 2008; 65: 28-37

48 Schultze-Lutter F, Ruhrmann S, Fusar-Poli P et al. Basic symptoms and the prediction of first-episode psychosis. Curr Pharm Des 2012; 18: $351-357$

49 De Herdt A, Wampers $M$, Vancampfort $D$ et al. Neurocognition in clinical high risk young adults who did or did not convert to a first schizophrenic psychosis: a meta-analysis. Schizophr Res 2013; 149: 48-55

50 Fusar-Poli P, Deste G, Smieskova R et al. Cognitive functioning in prodromal psychosis: a meta-analysis. Arch Gen Psychiatry 2012; 69: $562-571$ 